\title{
SPECTROSCOPIC STUDY OF POPULATION INVERSION MECHANISMS IN DIATOMIC-MOLECULE LASERS
}

\author{
L. Scaffardi, D. Schinca, J. O. Tocho, and H. F. Ranea-Sandoval \\ Centro de Investigaciones Opticas (CIOp), Casilla de Correo 124, 1900 La Plata (B.A.), Argentina
}

(Received 1 September 1987; received for publication 13 July 1988)

\begin{abstract}
Spectral analysis of laser radiation emitted by diatomic molecules and simple theoretical models have allowed us to study population-inversion mechanisms in the u.v. and i.r. for $\mathrm{N}_{2}$ and visible $\mathrm{CO}$ molecular lasers. The technique is readily applicable to other diatomic molecules. Observations of $\Lambda$-doubling in $\mathrm{N}_{2}$ u.v. laser emission and analysis of rotational structure in i.r. laser emission for the same molecule are used to determine the main features of electronic excitation and different channels for population of the laser levels involved. For the visible molecular $\mathrm{CO}$ laser, a fit of the rotational intensity distribution of the emitted visible bands was used to determine enhancement mechanisms by which population inversion is achieved in some $Q$-branch transitions.
\end{abstract}

\section{INTRODUCTION: OUTLINE OF THE METHOD}

An extensive review on $\mathrm{N}_{2}$ lasers was published recently.' Several questions remained unanswered concerning excitation mechanisms, particularly of levels involved in the first positive i.r. band system. In this paper, we present a method that can be applied to any diatomic molecule and allows determination of the relative importance of a proposed mechanism on population inversion between vibrational-rotational levels of different electronic states in the molecule. The method has been applied to the $\mathrm{N}_{2}$ and $\mathrm{CO}$ laser systems, using spectroscopic data derived from analysis of the rotational intensity distribution of the $0-1$ i.r. and $0-0$ u.v. bands in $N_{2}$ and of the $0-5,0-4$, $0-3$, and $0-2$ visible bands in $\mathrm{CO}$.

Two basic levels of information are needed. First, a high-resolution spectrum is required of the bands under study, from which the intensity distribution of the rotational lines can be reliably obtained. Second, a description is needed of the gain for each individual rotational line; this description is obtained from the theory of spectra for diatomic molecules, which is well established.

In writing the gain equation, proposed mechanisms for level population are included. We have studied participation of two different mechanisms in the population of levels, namely, direct impact excitation by slow electrons in a pulsed discharge and cascade population (and depopulation) by stimulated emission from upper levels.

Basically, we assume that, upon direct collisional excitation by slow electrons of molecules in the ground state, the rotational population is duplicated in the excited states, ${ }^{2}$ and there is no possibility for equilibration before the onset of stimulated emission because of the small ratio of radiative to collisional decay times. This approximation eliminates the need of knowing electron-molecule and molecule-molecule collision rates for time-dependent populations. Thus, population variation is adiabatically eliminated. For cascading, the use of optical-transition probabilities and statistical-weight factors allows us to model the process. If other population (depopulation) mechanisms are included, knowledge of their influence on rotational population redistribution is required.

With the specified assumptions, a simulated spectrum can be generated and fitted to observed data by varying parameters that measure the relative importance of different processes. A threshold-gain value is used to define the laser intensity of the simulated lines in the first approximation. Additional information on observed spectra may be used to reject mechanisms and to simplify the analysis, as will be shown for the case of $\Lambda$-doubling in the second positive system of $\mathrm{N}_{2}$. 
Three adjustable parameters must be chosen in our examples in order to fit a complete rotational spectrum. This requirement has proved to be highly restrictive and assures good accuracy for the parameters involved.

\section{$\mathrm{N}_{2}$ LASER}

Laser action in molecular nitrogen in the i.r. and u.v. was first obtained by Mathias and Parker ${ }^{3}$ and Heard, ${ }^{4}$ respectively. Since the lower level of the u.v. transition $\left(2^{+}\right.$system $)$is the upper level of the i.r. transition ( $1^{+}$system), as shown in Fig. 1, interaction between them may be assumed. Only a few studies deal with this interaction and there is some disagreement concerning the conclusions. ${ }^{5.6}$

Recently, Kruger ${ }^{7}$ has studied the interaction between the $1^{+}$and $2^{+}$systems by analysing the spontaneous sidelight emission from a discharge tube lasing in the i.r. He was able to reproduce the vibrational intensity distribution of the observed bands by varying a cascade factor $f$, related to stimulated emission connecting the $C^{3} \Pi_{\mathrm{u}}$ and the $B^{3} \Pi_{\mathrm{g}}$ states, in the rate equations. We have studied population mechanisms of the $C^{3} \Pi_{u}, B^{3} \Pi_{\mathrm{g}}$, and $A^{3} \Sigma_{\mathrm{u}}^{+}$levels by using a model that simulates the rotational intensity distribution of the $0-1$ i.r. laser band at different pressures. We compare this intensity distribution with experimental data.

Discharge tubes of different active lengths and internal diameters were used for both the axial and transverse excitation modes. The tubes were immersed in a liquid-air bath to increase the gain of the stimulated emission. A 3.4-m focal length, Ebert-mount spectrograph with a 600 grooves $/ \mathrm{mm}$ diffraction grating was used for the spectral analyses. Dispersions in the 1st and 9th diffraction order were 4.8 and $0.22 \AA / \mathrm{mm}$, respectively. A detailed description of the experimental setup has been given elsewhere. ${ }^{8.9}$

While nearly all laser bands present only one output maximum as a function of pressure, ${ }^{10}$ the $0-1$ i.r. band peaks at two different pressures (see Fig. 2). Since the pressure range used to study the $0-0$ u.v. band is the same as that for the low-pressure peak of the $0-1$ i.r. band, and the lower level of the u.v. band is the upper level for i.r. emission, it is plausible to assume that the population of the $v=0$ vibration level of the $B^{3} \Pi_{\mathrm{g}}$ state is due, in part, to a cascade process from the $C^{3} \Pi_{\mathrm{u}}$ state and, in part, to an electron-impact process. In this case, inhibition of u.v. emission should affect the low-pressure peak of the i.r. band.

It is apparent from Fig. 2 that inhibition of the feed-back of the u.v. radiation by suitable intracavity filters drastically reduces the intensity of the low-pressure peak of the $0-1$ i.r. band, which indicates that the proportion of the cascade population channelled by direct impact excitation is greater at lower pressures. This difference in excitation mechanisms affects the rotational structures of the emission spectra of both peaks, as is shown in Fig. 3. In order to

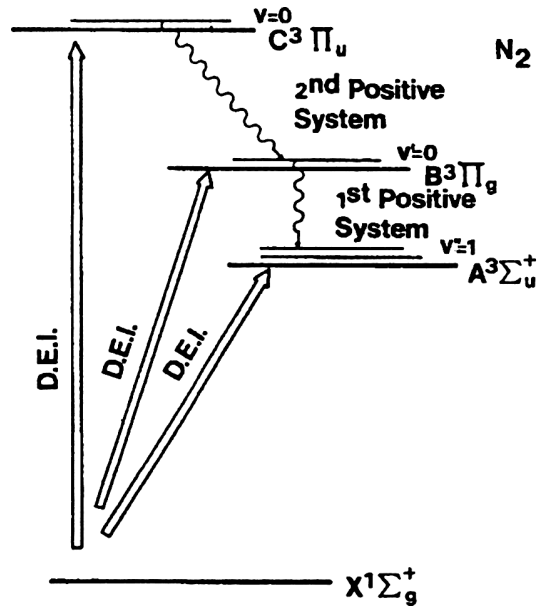

Fig. 1. Partial energy level diagram for a molecular laser.

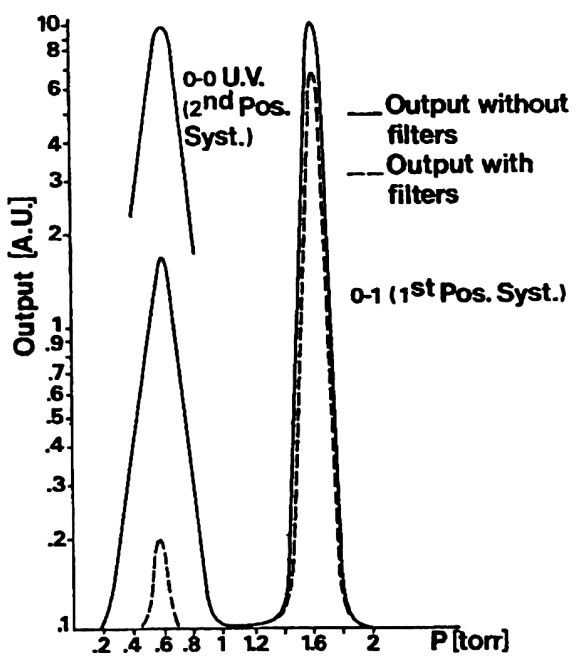

Fig. 2. Output of the $0-1$ i.r. laser band vs $\mathrm{N}_{2}$ pressure, with and without intracavity filters to block the $0=0$ u.v. laser band. 
simulate these rotational spectra, we assume that the $B^{3} \Pi_{\mathrm{g}}$ state population is caused, in part, by direct electron impact from the ground state and, in part, by radiative decay from the $C^{3} \Pi_{\mathrm{u}}$ state. The latter process selectively populates rotational levels of the $v=0$ vibrational level in the $B^{3} \Pi_{B}$ state, thereby destroying population equilibrium. This situation is stationary in the pressure range used since the collision frequency is not sufficiently high to redistribute the population during the time that i.r. laser emission lasts (150 ns). ${ }^{8}$

When a molecule is excited by electron collisions, large changes are not produced in the angular momentum of the system. " Therefore, molecules excited by electron impact from the ground state of the $B$-state show the same population distribution over the rotational level as in the initial state.

The population of the $B_{J}$ levels caused by cascading can be calculated by using optical selection rules governing the u.v. transitions $\left(C^{3} \Pi_{\mathrm{u}} \rightarrow B^{3} \Pi_{\mathrm{g}}\right)$ and a population for the $C$-state associated with pure electron impact. ${ }^{8}$ The specified rules only allow $P$ and $P^{\prime}(\Delta J=-1)$ or $R$ and $R^{\prime}(\Delta J=1)$ branches. Since only the $\Delta J=-1$ branches were observed in the u.v. laser spectra, we disregard the $R$ and $R^{\prime}$ branches in our analysis.

The population of rotational levels in the $v=0$ vibrational level of the $B$-state is given by

$$
N_{J}^{B}=\left[a_{1}(2 J+1)+a_{2} i\left(P, P^{\prime}\right)\right] 2^{n} \exp \left[-B_{x} J(J+1) h c / k T\right],
$$

where the first term in the bracket arises from electron impact and the second from cascading. Here, $a_{1}$ and $a_{2}$ are parameters that determine the ratio between cascading and direct impact. We normalize the population distribution by setting $a_{1}+a_{2}=1$ to obtain a purely thermal distribution for $a_{2}=0: J$ is the rotational quantum number, $B_{x}$ the vibrational constant of the ground state, $h$ Planck's constant, $c$ the velocity of light, $k$ Boltzmann's constant, $T$ the rotational temperature, $n$ an integer that has the values 0 or 1 (depending on whether the rotational level is asymmetric or symmetric, respectively), and $i$ the optical transition probability for the $P$ and $P^{\prime}$ branches given by Kovács ${ }^{12}$ when both states belong to Hund's case $a$ for small $J$ values. The population of the $A^{3} \Sigma_{u}^{+}$state may be assumed to be caused by electron impact from the ground state and is given by an expression similar to that for the $B$ and $C$ populations:

$$
N_{J}^{A}=(2 J+1) 2^{n} \exp \left[-B_{x} J(J+1) h c / k T\right] .
$$

The unsaturated gain $G_{J J}$ of laser lines is proportional to population inversion and to transition probability. Hence, we may write

$$
G_{J J^{\prime}}=\left[N_{J}^{B}-(1 / a)\left(g_{B} / g_{A}\right) N_{J}^{A}\right] i_{J J^{\prime}},
$$

where $a^{-1}=N_{v}^{A} / N_{v}^{B}$ is the inverse of the vibrational population inversion, $g_{B}$ and $g_{A}$ are the

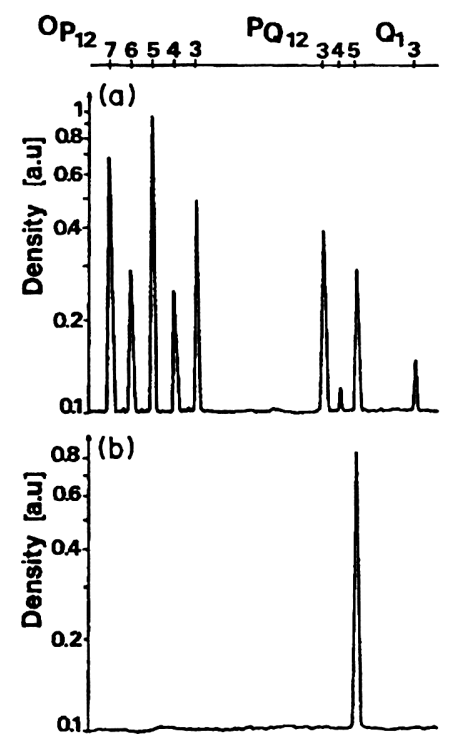

Fig. 3. Microdensitogram of (a) the high-pressure spectrum and (b) the low=pressure spectrum of the $0-1$ i.r. laser band.

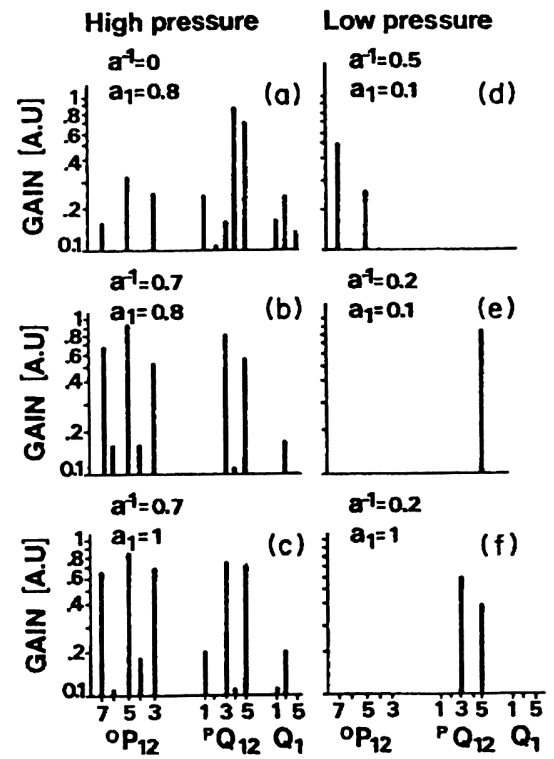

Fig. 4. Simulated spectra of the 0-1 i.r. laser band. 
statistical weights of the rotational level, and $i_{J J}$ is the optical transition probability for a given rotational transition when the $A$-state is in Hund's case $b$.

The relation between laser intensity and calculated gain was obtained by neglecting spontaneous emission. Above the threshold, the intensity is proportional to the gain, i.e., $I \propto\left(G-G_{\mathrm{T}}\right) .^{13}$ Thus, the intensity can be written as

$$
I= \begin{cases}0 & \text { if } G<G_{\mathrm{T}}, \\ 100\left(G-G_{\mathrm{T}}\right) /\left(1-G_{\mathrm{T}}\right) & \text { if } G \geqslant G_{\mathrm{T}},\end{cases}
$$

where $G$ is the gain that is normalized to the maximum unsaturated gain $G_{J J}$. Since we measure only relative intensities, this maximum was arbitrarily taken as 100 .

Using Eqs. (1)-(4), the intensity of the i.r. lines was calculated for different values of the parameters $a_{1}, a$, and $G_{\mathrm{T}}$. Some results are shown in Fig. 4. Figure 4(b) shows the simulated spectrum of the high-pressure peak for optimal values of $a_{1}$ and $a^{-1}$. It is possible to find a threshold value so that only lines with gains above this threshold can be observed experimentally.

It is, however, impossible to find such a value from Fig. 4(c) for which no cascading is assumed. Figure 4(a) shows that if the $A$-state population is neglected $\left(a^{-1}=-0\right)$, it is impossible to reproduce the high-pressure spectrum for any value of $a_{1}$. This result means that the dependence of the intensity distribution of the laser emission on $J$ does not follow the intensity distribution for spontaneous emission.

Similar results are shown for the low-pressure peak in Figs. 4(d)-(f). Unfortunately, the 0-1 i.r. band only lases in one line, a fact that prevents the accurate determination of best values for $a_{1}$ and $a^{-1}$. Nevertheless, these parameters can be determined to fit such a spectrum. The values for both parameters range from 0 to 0.4 , suggesting that there is a lower population of the $A$-state and a lower proportion of electron impact than in the high-pressure case.

Figure 4(e) shows a simulation of the low-pressure spectrum. However, if either $a \approx^{1}$ [Fig. 4(d)] or $a_{1}$ [Fig. 4(f)] are chosen beyond this range of values, the line of maximum intensity is not the experimentally observed curve.

The optimal parameters show that, in the low-pressure case, cascading contributes $70-90 \%$ to the population of rotational levels of the $v=0$ vibrational level of the $B$-state, while for the high-pressure case, this contribution amounts to $20 \%$. This result agrees with previously mentioned measurements of Kruger ${ }^{7}$ and with the results obtained through u.v. laser-radiation inhibition at low pressures.

\section{LAMBDA-DOUBLING}

The observation of the $\Lambda$-doublet components in transverse and longitudinal discharge spectra has led to clearer understanding of the excitation mechanisms of the $C$ - and $B$-states.

Massone et $\mathrm{al}^{8}$ were the first to observe both $\Lambda$-components of the $0-0$ u.v. laser band in axial-discharge lasers. The high resolution achieved in the spectra, together with a careful assignment of rotational lines, allowed the authors to discuss some previous conclusions given by Parks et al. ${ }^{14}$

The conclusions in Ref. 14 were that observation of only one of the $\Lambda$-components $\left(\Pi^{+}\right)$could be explained by a detailed analysis of the electron-collision process. For electron energies much greater than the excitation threshold and in the limit of small momentum transfer, the inelastic electron collision cross section can be expressed in terms of the optical transition probability factor. To show the general consistency of this approximation, the authors of Ref. 14 showed agreement between their calculated gain term and their observation of only one $\Lambda$-component.

Fortrat parabolas constructed in accordance with the results of Massone et al are shown in Fig. 5. The dots represent the values found by Parks et al, which were assigned by these authors to a single Fortrat parabola. Analysis of the experimental data of other authors and their own enabled Massone et al to show that the mean energy of electrons in the discharge should be close to the excitation threshold of the $C$-state $(11 \mathrm{eV})$, indicating that the assumption of high mean electron-excitation energy is not an appropriate mechanism for the $C$-state excitation.

Later work at our laboratory ${ }^{9}$ clearly showed the difference between the 0-0 u.v. laser band spectra obtained in axial and in transversed discharges (see Fig. 6), indicating that in the latter only 




Fig. 5. Partial Fortrat parabolas of the $P_{1}$ and $P_{1}^{\prime}$ branches of the $0-0$ u.v. laser bands. Dots represent the values assigned in Ref. 11 to a single parabola.

the strongest $\Lambda$-doublet components are observed. The authors ${ }^{9}$ concluded that the doublet may be observed if threshold excitation conditions are better met. This conclusion agrees with the results of Scaffardi et al, ${ }^{15}$ who observed both components in axial-type lasers, but only the alternating strong components in superfluorescent emission with the same geometry. The studies of RaneaSandoval et $\mathrm{al}^{16}$ on transverse discharges show growth of the weak $A$-components as the excitation increases. These results are in general qualitative agreement with those of Petit et al, ${ }^{17}$ as was noted in Ref. 18. Preliminary gain calculations assuming low mean electron-energy-excitation are in agreement with experimental observations of the $\Lambda$-doublets, both in axial and transverse discharges. For low-temperature axial lasers, the best fit of the observed intensity distribution was achieved for an $a$-value of 1.4; for transverse lasers at room-temperature, the best fit corresponds to an $a$-value of approx. 1.6.

\section{CO LASER}

In this section, we apply our procedure to the study of excitation mechanisms involved in the Angstrom system laser levels of the CO molecule.

Carbon monoxide laser action can be obtained in three spectral regions: the i.r. $(5 \mu \mathrm{m})$, the visible $(450-700 \mathrm{~nm})$, and the v.u.v. $(180-190 \mathrm{~nm})$. While the i.r. emission has been studied extensively (see, for example, Ref. 19), there are few studies dealing with visible ${ }^{20-23}$ and v.u.v. ${ }^{24}$ emission

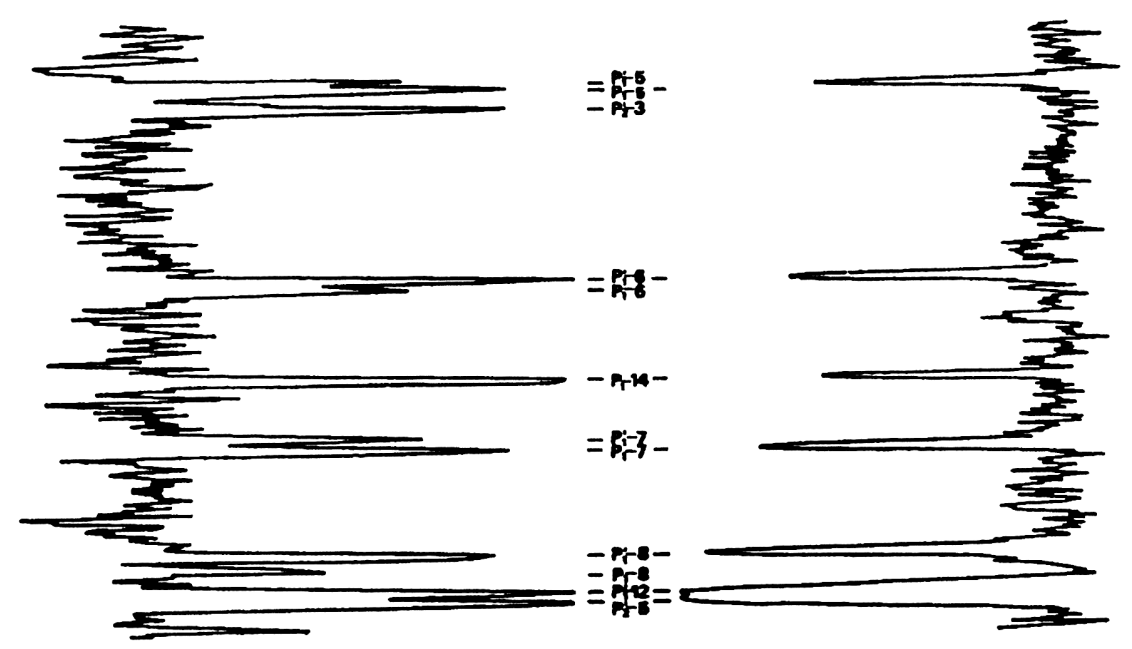

Fig. 6. Microdensitometric traces of a part of the $0-0(337.1 \mathrm{~nm}) \mathrm{N}_{2}$ laser band. The right=hand and left-hand traces correspond to axial and crossed field discharges, respectively. 

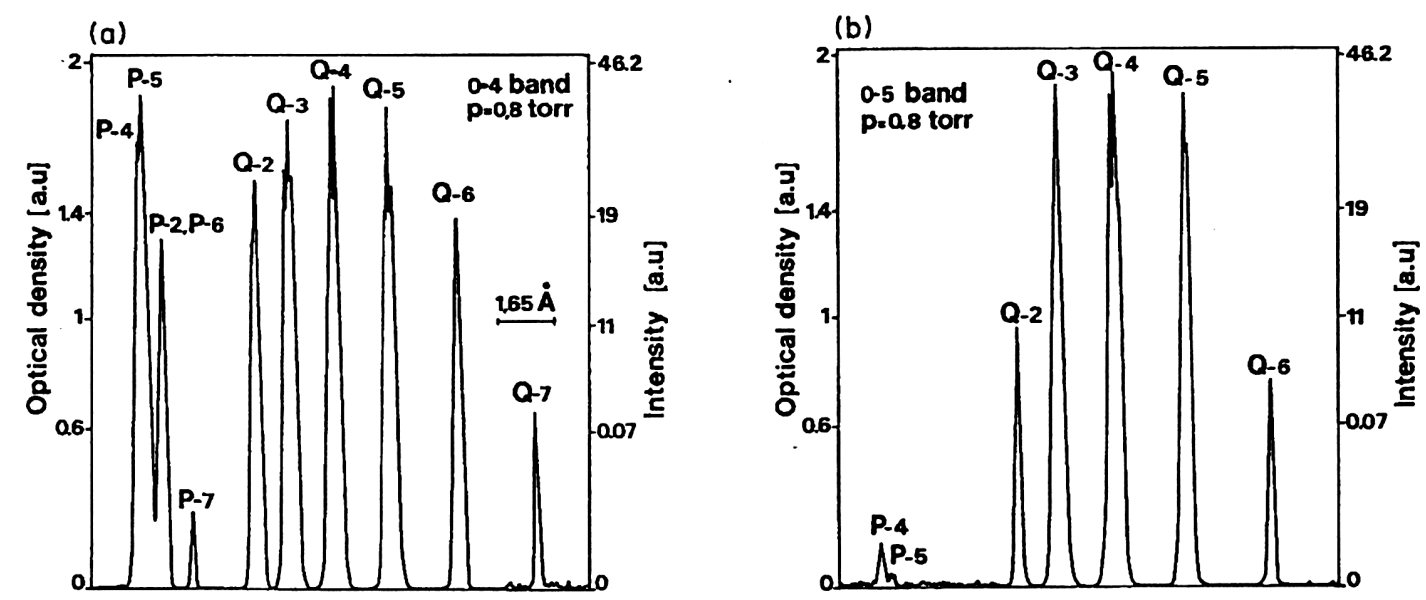

Fig. 7. Microdensitograms of the $\mathrm{CO}$ laser bands: (a) $0-4$ and (b) $0-5$. The dispersion is the same in both cases.

characteristics. Only one of these ${ }^{21}$ deals, in a preliminary way, with population inversion mechanisms of the Angstrom system.

In our experimental apparatus, commercial $\mathrm{CO}$ was allowed to flow slowly through an $80 \mathrm{~cm}$ long and $10 \mathrm{~mm}$ i.d. discharge tube, which was immersed in a liquid-air bath to increase the gain of the transitions. ${ }^{23}$ The spectrograph used was the same as that described in the section devoted to the $\mathrm{N}_{2}$ case. A detailed description of the experimental setup can be found elsewhere. ${ }^{25,26}$

Spectra of the 0-5, 0-4, 0-3, and 0-2 CO visible laser bands, at pressures ranging from 0.6 to 1.3 torr, were recorded. ${ }^{27}$ Figure 7 shows two microdensitograms of these bands at optimum pressure. The rotational structure of the bands consisted of only $Q$ - and $P$-branches. At low pressures, only $Q$-branch lines were observed.

Simulation of the observed spectra was carried out by calculating the gain of the electronic-vibrational-rotational transitions of interest, based on the following assumptions (see Fig. 8): (a) the $B^{\prime} \Sigma^{+}$state belongs to Hund's case $a . "$ This state is populated by direct electron impact (DEI) from the $X^{1} \Sigma^{+}$ground state. ${ }^{21}$ Because of its short lifetime $(25 \mathrm{~ns}),{ }^{28}$ the collision frequency for our pressure conditions is not high enough ${ }^{8}$ to equilibrate the population during the time the visible emission occurs (100 ns). Once again, the rotational population distribution duplicates that of the ground state and, therefore, the population of the rotational levels of the $B$-state may be written as

$$
N_{J}^{B}=N_{v}^{B}(2 J+1) \exp \left[-B_{x} J(J+1) h c / k T\right],
$$

where $N_{v}^{B}$ is the vibrational level population; (b) the $A^{\prime} \Pi$ state belongs to Hund's case $a$. " The

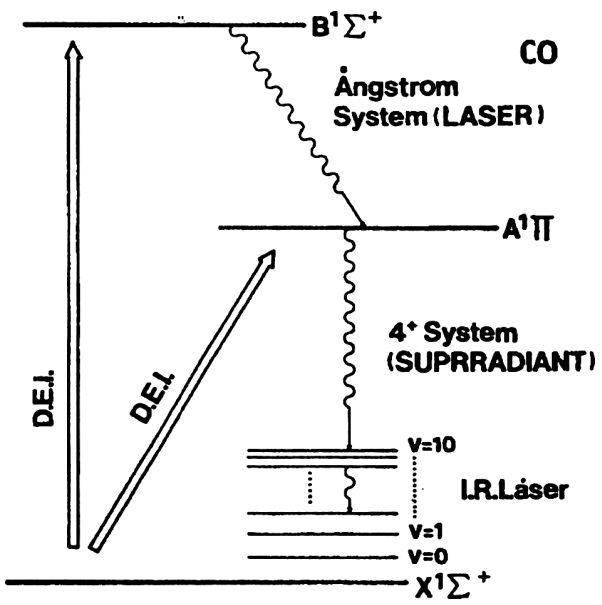

Fig. 8. Simplified energy level diagram of $\mathrm{CO}$. 
(a)

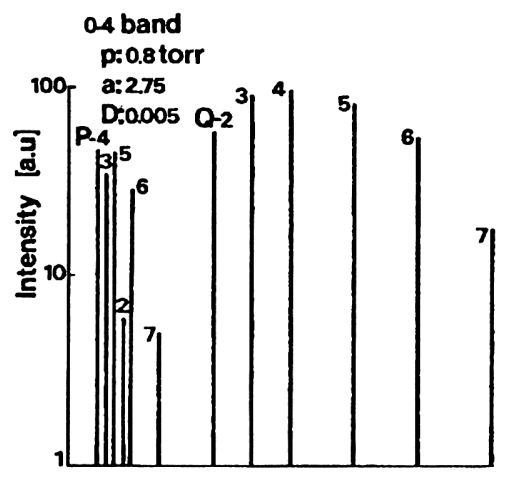

(b)

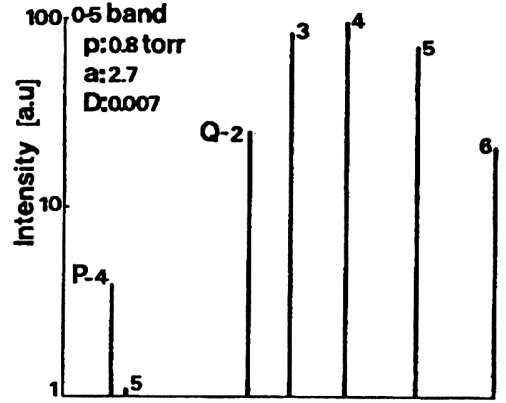

Fig. 9. Simulated spectra of the CO laser bands: (a) $0-4$ and (b) $0-5$.

$v=2,3,4,5$ vibrational levels are also populated by DEI from the ground state ${ }^{21}$ and duplicate the rotational population distribution because of the short lifetime of the rotational levels $(17 \mathrm{~ns}){ }^{29}$ Since the $A$-state is the upper level of the v.u.v. laser transition, it was assumed that its vibrational levels $v=2,3,4,5$, are depopulated by the v.u.v. radiative transition.

The fact that the v.u.v. laser bands have strong $Q$-branch, weak $R$-branch and no $P$-branch lines $^{24}$ suggests that there should be selective depopulation of $A$-state rotational levels, creating favourable conditions for population inversion of the $Q$-branch in the visible transition. Thus, the rotational population of the $A$-state can be written as a combination of DEI and radiative depletion, i.e.,

$$
N_{J}^{A}=N_{v}^{A}\left[(2 J+1) \exp \left[-B_{x} J(J+1) h c / k T-2 \Omega^{2}\right](1-2(J+1) D)\right],
$$

where $N_{v}^{A}$ is the vibrational level population of the $A$-state, $\Omega$ the total angular momentum projection, and $D$ the parameter measuring the porportion of v.u.v. emission. Introducing Eqs. (5) and (6) with the corresponding Hönl-London factor into Eq. (3), we write the gain for the $P, Q$, and $R$ branch as

$$
\begin{aligned}
G_{P}= & (1 / 2)(J+1) \exp \left[-B_{x} J(J-1) h c / k T\right]-(1 / a) \exp \left[-B_{x} J(J+1) h c / k T-2\right], \\
G_{Q}= & (1 / 2)(2 J-1) \exp \left[-B_{x} J(J+1)\right] \\
& -(1 / a)(1-D(2 J+1)) \exp \left[-B_{x} J(J+1) h c / k T-2\right], \\
G_{R}= & (1 / 2) J \exp \left[-B_{x}(J+1)(J+2) h c / k T\right]-(1 / a) \exp \left[-B_{x} J(J+1) h c / k T-2\right],
\end{aligned}
$$

where $J$ is the rotational quantum number of the $A$-state, and $a=N_{v}^{B} / N_{v}^{A}$ is the vibrational population inversion ratio.

The observed spectra were simulated by introducing Eqs. (7)-(9) in Eq. (4) and varying the parameters $a, D$, and $G_{\mathrm{T}}$. A weighted least-squares method was used in this simulation. The calculated spectra are shown in Fig. 9 and correspond to those of Fig. 7. In each case, the optimal

(a)

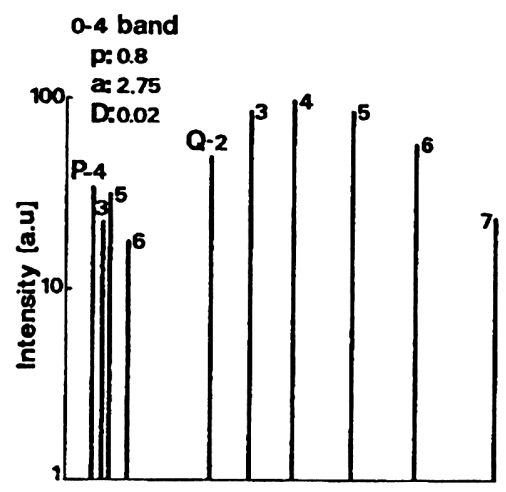

(b)

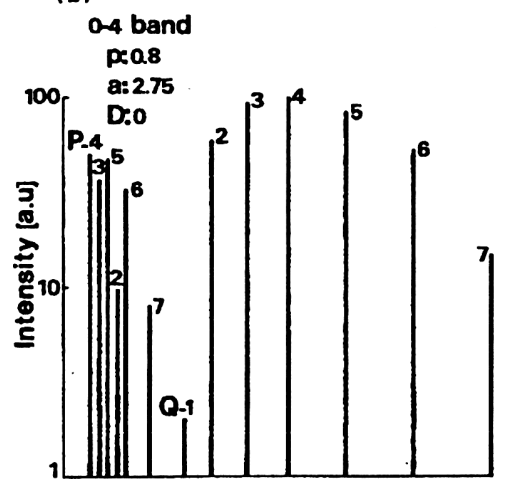

Fig. 10. Simulated spectra of the 0-4 CO laser band for non-optimal $a$ and $D$ parameters.

Q.S.R.T. 41 ? 
values of $a$ and $D$ are indicated. For the $0-4$ and $0-5$ bands, $D$ increases rapidly with pressure, suggesting that depopulation of the lower level enhances the gain of the bands at high pressure.

Simulated spectra calculated for other values of $a$ and $D$ are shown in Fig. 10. For the $0-4$ band, a $D$ value above the optimal estimate makes the $P-2$ and $P-7$ lines disappear [Fig. 10(a)], while a $D$ value below the optimal results in the appearance of the $Q-1$ line [Fig. 10(b)]. The method is very sensitive to small changes of $a$ and $D$, a fact that enables us to obtain an accurate simulation of spectra showing different rotational structures.

These results suggest a strong contribution involving cascading from the $4^{+}$system to the visible transition gain, especially in the $0-4$ and $0-5$ bands. This enhancement of the gain of the $Q$-branch lines of the visible spectra is produced by selective depletion of the lower laser level through v.u.v. radiative transitions.

\section{CONCLUSIONS}

Our analysis shows that it is possible to establish quantitatively excitation mechanisms that lead to population inversion in some transitions by using a simple theoretical model that reproduces the spectral intensity distribution. This method is particularly useful for diatomic molecules, for which the large number of rotational lines provides a good deal of information for an accurate fitting of the spectra. The procedure proved to be extremely sensitive to variations of the fitting parameters. Small deviations from optimal values distort the simulated intensity distribution in such a way that it is impossible to reproduce the observed spectra. The assumptions made about populations of the involved levels on excitation simplify gain-theoretical calculations and proved to be a useful approximation for diatomic molecules.

\section{REFERENCES}

1. R. S. Kunabenchi, M. R. Gorbal, and M. I. Savadatti, Prog. Quant. Electron. 9, 259 (1984).

2. V. N. Ochkin, S. Yu. Savinov, and N. N. Sobolev, Soviet Phys. JETP 48, 232 (1978).

3. L. E. S. Mathias and J. T. Parker, Appl. Phys. Lett. 3, 16 (1963).

4. H. G. Heard, Nature, Lond. 200, 667 (1963).

5. L. Allen, D. G. C. Jones, and B. M. Sivaram, Phys. Lett. A 25, 280 (1967).

6. M. Garavaglia, M. Gallardo, and C. A. Massone, Phys. Lett. A 28, 787 (1969).

7. J. S. Kruger, Ph.D. Thesis, The American University, Washington, DC (1981).

8. C. A. Massone, M. Garavaglia; M. Gallardo, J. A. Calatroni, and A. A. Tagliaferri, Appl. Opt. 11, 1317 (1972).

9. J. O. Tocho, H. F. Ranea-Sandoval, A. A. Tagliaferri, M. Garavaglia, M. Gallardo, and C. A. Massone, Nouv. Rev. d'Opt. 5, 319 (1974).

10. L. Scaffardi, D. Schinca, J. O. Tocho, H. F. Ranea-Sandoval, and M. Gallardo, Appl. Opt. 24, 22 (1985).

11. G. Herzberg, Molecular Spectra and Molecular Structure-I. Spectra of Diatomic Molecules, Van Nostrand-Reinhold, Princeton, NJ (1950).

12. I. Kovács, Rotational Structure of the Spectra of Diatomic Molecules, Adam Hilger, London (1969).

13. A. Yariv, Quantum Electronics, 2nd edn, Chap. 9, Wiley, New York, NY (1975).

14. J. H. Parks, D. Ramachandra Rao, and A. Javan, Appl. Phys. Lett. 13, 142 (1968).

15. L. Scaffardi, D. Schinca, J. Reyna-Almandos, M. Gallardo, and M. Garavaglia, Appl. Opt. 19, 3590 (1980).

16. H. F. Ranea-Sandoval, J. O. Tocho, M. Gallardo, and M. Garavaglia, IEEE J. Quantum Electron. QE-17, 295 (1981)

17. A. Petit, F. Launay, and J. Rostas, Appl. Opt. 17, 3081 (1978).

18. H. F. Ranea-Sandoval, J. O. Tocho, M. Gallardo, and M. Garavaglia, Appl. Opt. 18, 1705 (1979).

19. R. M. Osgood, Jr., W. C. Eppers, Jr., and E. R. Nichols, IEEE J. Quantum Electron. QE-6, 145 (1970) and references therein.

20. L. E. S. Mathias and J. T. Parker, Phys. Lett. 7, 194 (1963).

21. M. G. Cooper and P. K. Cheo, Appl. Phys. Lett. 5, 44 (1964).

22. A. Henry, G. Arya, and L. Henry, C. R. Acad. Sci. 261, 1465 (1965).

23. V. M. Kaslin and G. G. Petrash, Proceedings P. N. Lebedev Physics Institute, N. G. Basov ed., Moscow (1975).

24. R. T. Hodgson, J. Chem. Phys. 55, 5378 (1971).

25. D. Schinca, J. O. Tocho, L. Scaffardi, and M. Gallardo, Optica Pura Apl. (Spain) 18, 211 (1985). 
26. D. Schinca, H. F. Ranea-Sandoval, L. Scaffardi, and J. O. Tocho, IEEE J. Quantum Electron. QE-22, 2272 (1986).

27. D. Schinca, L. Scaffardi, and J. O. Tocho, Appl. Opt. 25, 102 (1986).

28. J. Rogers and R. Anderson, JOSA 60, 278 (1970).

29. J. C. Chervenalk and R. Anderson, JOSA 61, 952 (1971). 